\title{
El usuario en el diseño de productos y servicios
}

Leidy Carolina Soto Cárdenas*

DOI: https://doi.org/ 10.33571/revinterseccion.v2n4a5

\section{Resumen}

Cuando se hace referencia al "usuario" se habla de aquella persona que utiliza algún artefacto para una función específica. El término es genérico y se limita en primera instancia a describir la acción de una persona que usa algo. Este artículo se pregunta ¿cómo se debería definir el concepto de usuario para un diseñador? Para ello aborda lo referente a las experiencias y emociones, customer journey, el persotipo y el diseño de experiencia en empaques.

Palabras claves: Usuario, customer journey; persotipo; diseño; empaques; cliente; servicios.

Recibido. Octubre 23, 2020 Aceptado. Noviembre 10,2020

*Diseñadora Industrial y Diseñadora de Vestuario de la Universidad Pontificia Bolivariana - Sede Medellín, Desarrolladora de nuevos productos en prozyectos de empaque. Orcid: https://orcid.org/0000-0001-9411-3994; e-mail: leidycarolinasoto@ hotmail.com 


\title{
The user in the design of products and services
}

\author{
Leidy Carolina Soto Cárdenas*
}

DOI: https://doi.org/ 10.33571/revinterseccion.v2n4a5

\section{Abstract}

When we refer to the "user" we allude to that person who makes use of a device for a specific function. It is necessary that the user is aware that what he is doing has a logical and specific purpose, in the first instance, the term is generic and limited to describe the action of a person who uses something. This article enquires, how should the user concept be defined for a designer? To that end, it deals with what experiences and emotions mean, customer journey, the persotype and packaging experience design.

Keywords: User, customer journey; persotype; design; packaging; customer; services. 


\section{O Usuário no projeto de produtos e serviços}

Leidy Carolina Soto Cárdenas*

DOI: https://doi.org/ 10.33571/revinterseccion.v2n4a5

\section{Resumo}

Quando nos referimos ao "usuário", estamos falando daquela pessoa que usa algum dispositivo para uma função específica. É necessário que ele esteja consciente de que o que ele está fazendo tem um propósito lógico e conciso, no entanto, o termo é genérico e limitado em primeira instância para descrever a ação de uma pessoa que usa algo. Este artigo se pergunta -como o conceito de usuário deve ser definido para um projetista? Para fazer isso, aborda experiências e emoções, a viagem do cliente, o persotipo e o projeto da experiência em embalagens.

Palavras chave: Usuário, viagem do cliente; persotipo; projeto; embalagem; cliente; serviços.

Recebido. Outubro 23, 2020 Aceitado. Novembro 10 de 2020

*Projetista Industrial e Projetista de Figurinos da Universidad Pontificia Bolivariana - Sede Medellín, Desenvolvedora de novos produtos em projetos de embalagem. Orcid: https:// orcid.org/0000-0001-9411-3994 ; e-mail: leidycarolinasoto@hotmail.com 


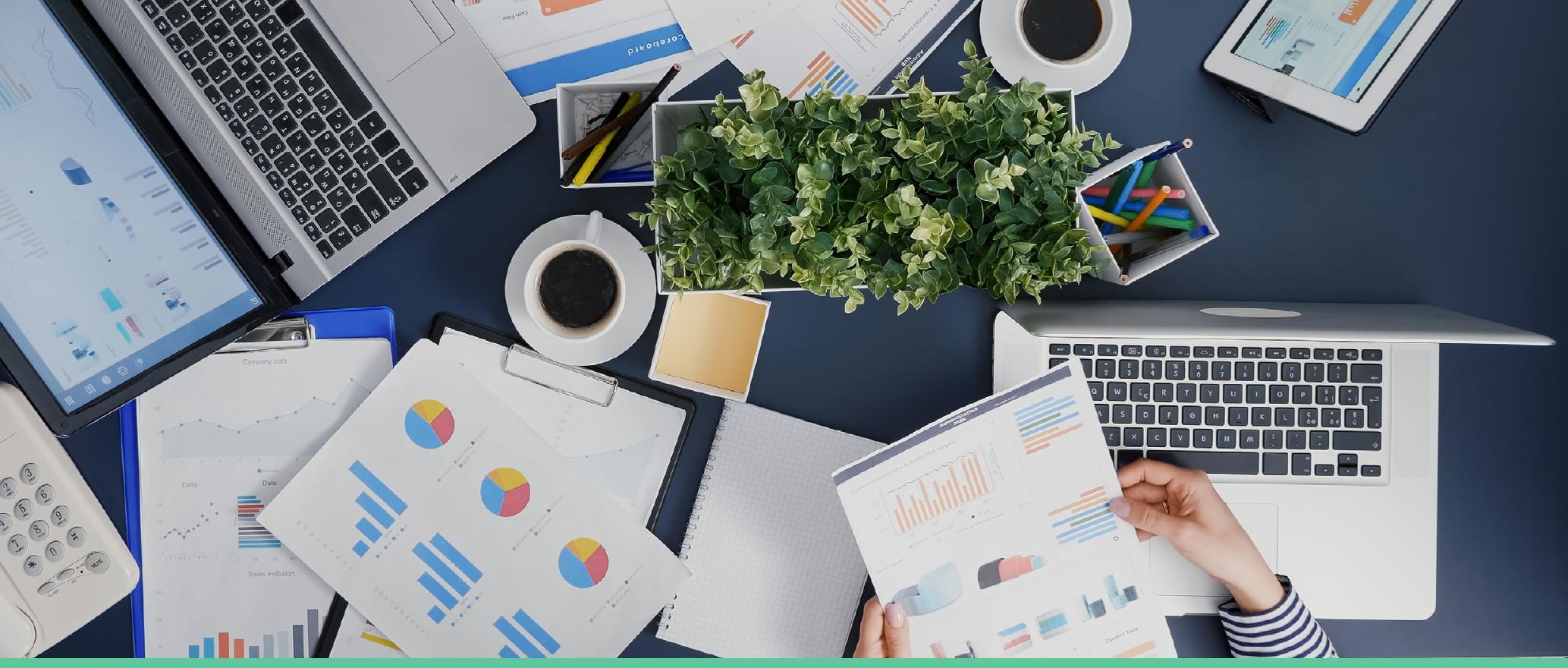

\section{Introducción}

radicionalmente se ha analizado cada una de las características funcionales de los productos, desde la elección de los materiales, los procesos y la relevancia funcional de estos, pero ż dónde tiene lugar el usuario, el actor principal en todo este proceso? ¿cómo se debería definir el concepto de usuario para un diseñador? Esta pregunta es el eje fundamental del diseño, para descifrar que un producto por sí solo no tiene ningún valor funcional y emocional, si no tiene un vínculo con el sujeto al que pertenece la experiencia de uso.

Es realmente difícil definir este término, ya que cada uno se construye de necesidades y deseos acordes al contexto, la cultura y las creencias en las que se desarrollan; aun así, podría definirse como un ser capaz de razonar y crecer, como ser pensante y emocional, y esto es debido a la evolución de nuestro cerebro y su capacidad de alcanzar diferentes niveles de intelecto; a esto se le suma la interacción de un usuario con un producto $u$ objeto en concreto, creando el término, que relaciona el uso de un artefacto con el vínculo emocional creado por el sujeto.

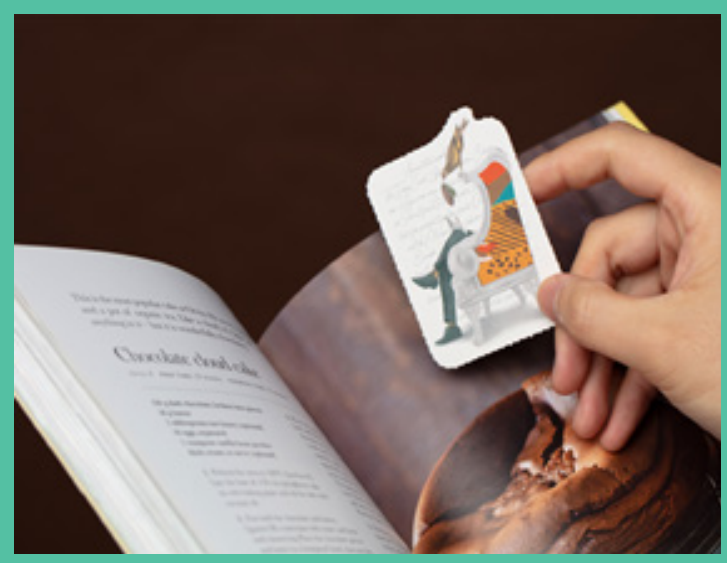


Como se menciona en el texto Product Attachment: Design Strategies To Stimulate The Emotional Bonding To Products (2008) estos enlaces a los productos es mera cuestión de agrado, las posesiones favoritas de las personas están relacionadas con los nexos emocionales más fuertes; el objeto de apego al que la persona se une, genera emociones positivas con relación a este, comprende sensaciones de felicidad, comodidad, sorpresa, entre otras, pero los objetos no son los únicos que pueden generar experiencias, también pueden ser los espacios, las personas y los momentos, es por esto que una experiencia no debe ser entendida por sí sola, separada de los demás elementos del entorno, ya que cada una de las situaciones externas o internas al sujeto influencian en como éste percibe su interacción con el producto.

En ocasiones el individuo, puede proporcionar un significado a un objeto meramente utilitario, puede considerarlo funcional o práctico, pero el apego a un objeto en específico trasciende del mero uso de este; combinando aspectos tanto emocionales como funcionales. Desde el diseño de producto, el diseñador es quien debe entender cada una estas interacciones, fortaleciendo el vínculo emocional entre el individuo y el producto.

La creación de experiencias emocionales puede ser un factor decisivo de compra para el sujeto y una ventaja competitiva para el diseñador, como se habla en el artículo de Nuevas tendencias del marketing: El Marketing Experiencial de Entelequia (2012).

\begin{abstract}
"El incremento de la competencia en los mercados y la existencia de una mayor competitividad entre las empresas, así como los cambios en el comportamiento de compra de los consumidores ha puesto de manifiesto la necesidad de adoptar una concepción del marketing más adaptada a la realidad actual del mercado y de las demandas de los consumidores (Palmer, 2010, Srinivasan y Srivastava, 2010).
\end{abstract}

Así surge el denominado Marketing Experiencial, uno de los últimos avances de la disciplina del Marketing, que se dirige a ofrecer al consumidor una experiencia de consumo única, estableciendo una vinculación con la marca o empresa, basada en las emociones, sentimientos o pensamientos, entre otros aspectos, que despierta el producto en el consumidor." (M. Moral, M. Alles. 2012)

Un producto o servicio puede tener lo necesario para satisfacer una necesidad o mejorar una experiencia, pero si el usuario no comprende su uso o intención, este finalmente no tendrá ninguna relevancia. Es por esta razón, que el diseñador debe tener en cuenta los affordances, entendido este como la percepción e interacción del entorno del comprador con el producto. "Los clientes son individuos racionales y emocionales. Basan sus decisiones en estímulos sensoriales, desean verse estimulados y provocados de un modo creativo." (M. Moral, M. Alles. 2012).

Por otro lado, un producto puede ofrecer una experiencia para un usuario al presentar ciertas características que contribuyan al cumplimiento de una necesidad psicológica básica de este. Para percibirlo, el usuario debe entender por completo el funcionamiento del producto o tener una idea básica de su uso. Además, vale la pena señalar que la percepción de experiencia a partir de los Affordances no está vinculada solo a cómo un usuario puede lograr entender una característica experiencial, sino que también tiene que ser entendido con respecto a la disposición del usuario hacia dicha experiencia. Cuando el cliente no comprende del todo su uso y funcionamiento, este puede ignorarlo fácilmente. 
Es importante incluir el espacio dentro de un diseño de experiencia, también llamado diseño sensorial, relacionado directamente con la atmósfera del servicio prestado, este implica el diseño de entornos para producir efectos emocionales específicos en el comprador de modo que la experiencia de compra sea satisfactoria. Los cinco sentidos (vista, sonido, olfato, gusto y tacto) se consideran cruciales para el diseño de elementos tangibles en servicios centrados en la experiencia.

Pero como menciona Rodrigo Ronda León en su artículo Diseño de Experiencia de Usuario: etapas, actividades, técnicas y herramientas, "... no existe nada absoluto, las decisiones de diseño más acertadas varían en función del contexto, los contenidos y los usuarios. Es por eso que resulta difícil definir pautas o metodologías rígidas para su realización, y se hace imprescindible que los diseñadores afronten de forma flexible cada proyecto concreto" (2013).

\subsection{Diseño de experiencia en empaques}

Se sabe que actualmente este conocimiento sobre experiencias es utilizado por múltiples compañías para establecer relaciones comerciales duraderas, pero como se menciona en el libro Data Elevates The Customer Experience (2016) para que una experiencia sea exitosa se requieren nuevas formas de explorar las tendencias, preferencias de los clientes, y ser más inteligente sobre la respuesta a estos factores, teniendo en cuenta que cada contexto presenta necesidades diferentes y características sociales y culturales distintas, cada proceso investigativo debe arrojar insights relacionados con las emociones de los clientes y estos deben arrojar a su vez decisiones desde el diseño. Un Insight es uno de los términos más usados en el contexto del Marketing digital, es una clave, la esencia que nos permite encontrar la solución a un problema. Un camino, un dato que nos sugiere como resolver cualquier ecuación por compleja que sea. Así que las ventajas de aplicar insights en las estrategias de Marketing son muchas y muy diversas. Gracias a esto logramos alimentar nuestro producto añadiéndole las demandas y necesidades que el usuario tenía antes ocultas y que desconocíamos.

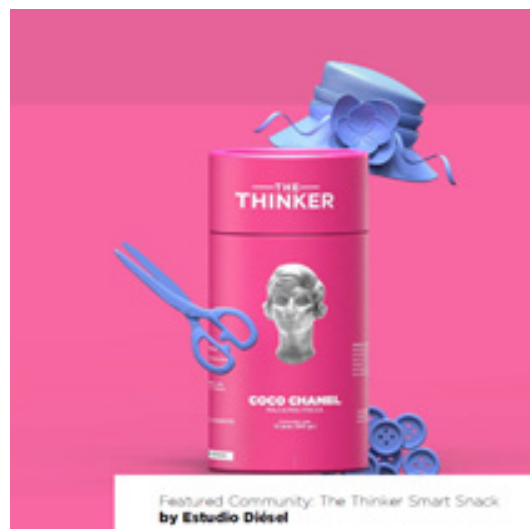

Aunque estos últimos campos se relacionan de manera directa con servicios, esto no quiere decir que la interacción no pueda presentarse en productos tangibles, como lo son los empaques; para ello es necesario definir el término empaque como contenedor colectivo que unifica y tiene como función proteger y contener el producto durante la etapa de distribución; adicional a esto, las compañías comprenden los empaques como la herramienta que se encuentra en sintonía con las exigencias del mercado y es la primera oportunidad que se tiene con el cliente por lo que debe ser definitiva para adquirir el producto.

Desde comienzos del 2020, todas las industrias se han transformado, aplicando diferentes estrategias de innovación, para la conversión de sus productos en el mercado; según la editorial La República, el comercio electrónico ha crecido más de $300 \%$ en Latinoamérica en la pandemia. Como lo menciona Marcos Pueyrredon, presidente del InstitutoLatinoamericano de Comercio Electrónico (eCommerce Institute)

"El avance que ha registrado el sector a nivel regional se debe a la continuidad de la operación de los retailers y marcas que tenían desarrollados sus capacidades digitales y a que todo lo que se hacía en forma física o presencial no tuvo más remedio que virtualizarse o digitalizarse" 
Así que ¿̇cómo se reinventa la industria de los empaques en un nuevo mercado donde prevalecen los e-Commerce? Anteriormente, miles de productos competían en los mercados, buscando ser adquiridos por los consumidores a través de estrategias de merchandising; pero el panorama ha cambiado y la respuesta frente a esta nueva transformación recae sobre el diseño de experiencias, cuya práctica resulta ser exitosa para aquellas empresas que desean generar valor emocional en sus consumidores.

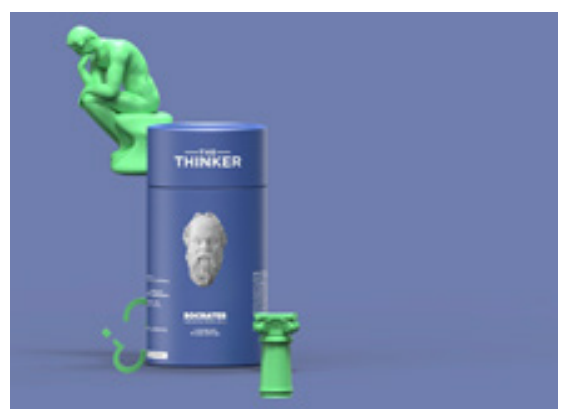

Para lograr generar nuevas experiencias de consumo, debe tenerse a consideración la fase de investigación en el proceso de innovación en experiencias, es decir la investigación centrada en los deseos del usuario. Cuando se habla de deseo del usuario nos referimos al análisis de necesidades o deseos presentes en los consumidores finales, los cuales serán la base de desarrollo para tangibilizar las ideas conceptualizadas; si se tiene en cuenta la nueva configuración descrita anteriormente, encontraremos que el

proceso investigativo no estará centrado en los tradicionales puntos de venta, sino que por el contrario las plataformas digitales y las condiciones de recepción de los productos harán definitiva la experiencia de consumo, es por esto que para generar un proceso exitoso se deben analizar más a profundidad como se vive la experiencia de apertura de los empaques en los hogares y como se desempeña la marca en estos contextos de uso, para ello pueden formularse algunas preguntas como:

¿qué sentimientos evoca el empaque? ¿Está el empaque aportando a la mejora en la experiencia de consumo? ¿ Cuál es la vida útil del empaque y que función cumple en el post consumo? ¿Qué aspectos sensoriales se están aplicando al empaque? Como se ha mencionado estas preguntas están orientadas a visualizar los productos más allá de artefactos funcionales y su intención se concentra en generar nuevos cuestionamientos alrededor de que es un producto.

\section{Conclusión}

Diseñar una experiencia abarca numerosas interacciones y encierra diferentes términos, pero el más importante de ellos es el "usuario" como actor principal de este proceso. El diseño de experiencia, es un punto de partida, es una herramienta que permite la creación de productos centrados en personas para personas; "...que más importante que la generación de productos, el diseño realmente debe producir significados que estén orientados a mejorar la calidad de vida y facilitar la interacción entre las personas" (Juan Camilo Buitrago. (2013). Diseño y producto. Santiago de Cali).

Con el entendimiento de este concepto, se establece un enfrentamiento con la tendencia de producción en masa de objetos que no poseen ningún valor emocional para el individuo, objetos que han pasado de ser particulares a generales y se ha perdido la creación de productos con significados que transcienden el mero uso de estos; pero no todo está perdido, son los diseñadores y empresarios quienes pueden marcar la diferencia entendiendo a los seres humanos como aquello que son y no como parte de una cadena de consumo.

"proponer modelos donde prime la identificación del ser humano como poseedor de características propias, irrepetibles, necesarias de ser atendidas y potencializadas mediante objetos realmente únicos que reflejen su carácter individual" (Miguel Uribe. (2013). Diseño y producto. Santiago de Cali). 


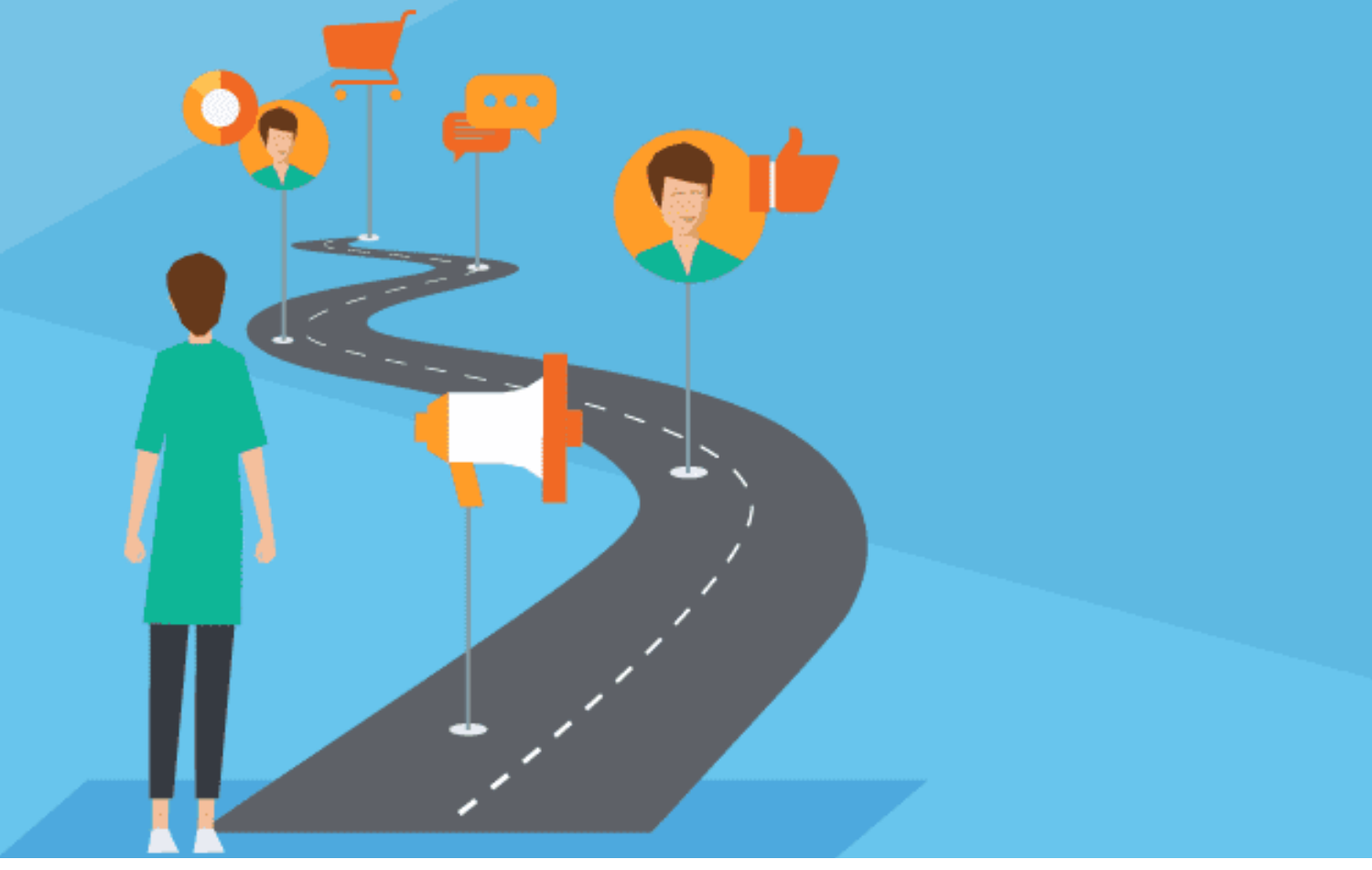

\section{Referencias Bibliográficas}

A. Solórzano, M. Uribe, M. González. Santiago de Cali, Julio de (2013). Diseño y producto. Facultad de Artes Integradas Universidad del Valle. Plancha para impronta No. 4

F. Pucillo and G. Cascini. (2014) . A framework for user experience, needs and affordances. Elsevier Ltd.

G. Zomerdijk, Leonieke, Voss, Chris (2010). Service Design for Experience-Centri Services. Journal of Service Research - J SERV RES. VL - 13. https://www.larepublica.co/globoeconomia/ e-commerce-ha-crecido-mas-de-300-en-latinoamerica-en-medio-de-la-pandemia-3000424.

M. Moral, M. Alles. (2012). Nuevas Tendencias Del Marketing: El Marketing Experiencial.

Entelequia. Revista interdisciplinar -Núm. 14.Disponible en: https://www.researchgate. net/profile/Maria-Teresa-Alles/publication/243056539_El_marketing_experiencial_ antecedentes_y_estado_actual_de_la_investigacion/links/0deec538359e2019a3000000/Elmarketing-experiencial-antecedentes-y-estado-actual-de-la-investigacion.pdf

R. Mugge, J. P.L. Schoormans, H. Schifferstein. (2008). Product Attachment: Design Strategies To Stimulate The Emotional Bonding To Products. Elsevier Ltd. Disponible en : https://www.academia.edu/14973255/Product_attachment_Design_strategies_to_stimulate_ the_emotional_bonding_to_products

Data Elevates The Customer Experience (2016). Disponible en: https://www.sas.com/content/ dam/SAS/en_us/doc/whitepaper2/forbes-data-elevates-customer-experience-108235.pdf

Para citar este artículo

Soto, L. (2020). El usuario en el diseño de productos y servicios. Revista Intersección: Eventos, Turismo, Moda y Gastronomía. Vol. 2 Núm. 4. Pp: 63-71; https://doi.org/ 10.33571/revinterseccion.v2n4a5

OJS: https://revistas.elpoli.edu.co/index.php/int/issue/archive Link: https://www.politecnicojic.edu.co/index. pho/revista-interseccion 